This article was published in Physical Chemistry Chemical Physics, 16 (31), 1651516523,2014

http://dx.doi.org/10.1039/c3cp55473b

\title{
Hematite Photoelectrodes for Water Splitting: Evaluation of the Film Thickness Role by Impedance Spectroscopy
}

Tânia Lopes ${ }^{1}$, Luísa Andrade ${ }^{1}$, Florian Le Formal, ${ }^{2}$ Michael Gratzel, ${ }^{2}$ Kevin Sivula, ${ }^{2}$ Adélio Mendes ${ }^{1 *}$

${ }^{1}$ LEPABE - Faculdade de Engenharia, Universidade do Porto, rua Dr. Roberto Frias, 4200-465 Porto, Portugal

2 Institute of chemical sciences and engineering, École Polytechnique Fédérale de Lausanne, Station 6, CH-1015 Lausanne, Switzerland

* Corresponding author: Tel.: +351 225081695; Fax: +351 225081449; E-mail address: mendes@fe.up.pt

\begin{abstract}
The electrochemical behavior of $\alpha-\mathrm{Fe}_{2} \mathrm{O}_{3}$ photoelectrodes prepared by spray pyrolysis with different thicknesses was examined under dark and illumination conditions. The main charge transport phenomena occurring in the PEC cell photoelectrodes were characterized by electrochemical impedance spectroscopy (EIS) operating under dark conditions. The impedance spectra were fitted to an equivalent electrical circuit model for obtaining relevant information concerning reaction kinetics and charge transfer phenomena occurring at the semiconductor/electrolyte interface. A three-electrode configuration was used to carry out the electrochemical measurements allowing a detailed study concerning the double charged layer at the semiconductor/electrolyte interface that arises under dark conditions. The model parameters determined by EIS were then related with film thickness to assess the role of electronic conduction on the performance of the cell. Moreover, correlating the samples thickness differences with their electrochemical impedance spectroscopy response, it was possible to discriminate the two main phenomena occurring on semiconductor/electrolyte interfaces of photoelectrochemical systems under dark conditions: the space charge layer and the electrical double layer.
\end{abstract}

Keywords: Solar Fuel, Water splitting, Hydrogen, Hematite, Electrochemical Impedance Spectroscopy, Electrical Analogues. 


\section{Introduction}

Hydrogen production by water splitting using solar energy is one of the most ambitious visions to overcome fossil fuel dependence in a sustainable way. Nowadays, the best performing materials for water photoelectrolysis are metal-oxide semiconductors since they are chemically more stable. ${ }^{1-3}$ However, despite the great efforts developed since the pioneer work by Fujishima and Honda in the 1970s for designing suitable materials, none of the available metal-oxide semiconductors can promote alone water splitting with a significant efficiency. ${ }^{4}$ Water splitting in a photoelectrochemical cell is determined by several key parameters, which must be simultaneously fulfilled: i) the photoelectrode has to have strong light absorption in the visible spectrum, ii) high chemical stability in aqueous electrolyte solutions under dark and illuminated conditions, iii) suitable band edges positions for hydrogen and oxygen evolutions, iv) low kinetic overpotentials and finally v) the charge transfer at the semiconductor/electrolyte interface must be selective for water splitting. ${ }^{1,2}$ The phenomena occurring at the semiconductor/electrolyte interface are rather complex and they are not yet fully understood despite their great importance on PEC cells performance. ${ }^{5}$

Electrochemical impedance spectroscopy (EIS) is a powerful technique to characterize the electrical behaviour of the semiconductor/electrolyte interface determined by a number of individual processes, most of them proceeding at different rates. ${ }^{6}$ This is a modulation technique where a small sinusoidal perturbation on the potential is applied to the system and the amplitude and phase shift of the resulting current response are measured. Moreover, the analysis of EIS spectrum provides information about the structure of the electrical double layer at the semiconductor/electrolyte interface and the charge transport processes occurring in the semiconductor and in the electrolyte. ${ }^{2,5,7-9}$. Despite being a relatively easy method to apply to the study of electrochemical systems, impedance spectra are sometimes difficult to be interpreted. The use of equivalent electrical analogues to fit the EIS experimental data is a very useful tool as it helps to identify and interpret some of the characteristic parameters of the system.

In the present work a simple electrical analogue is developed for correlating the phenomena occurring in the semiconductor/electrolyte interface, as well as in the semiconductor bulk. The proposed electrical analogue was validated based on $\alpha-\mathrm{Fe}_{2} \mathrm{O}_{3}$ photoelectrodes prepared by spray pyrolysis. 


\section{Experimental}

Films of hematite were prepared as described elsewhere. ${ }^{10}$ Iron (III) acetylacetonate was sprayed to a tetraethoxysilicate (TEOS)-pretreated substrate of FTO (glass $30 \mathrm{~mm} \times 12$ mm; TEC 15, Hartford Glass Co.; $15 \mathrm{~V}$ square $^{-1}$ ). The spray setup consisted of an ultrasonic spray head (Lechler company, US130 $0^{\circ}$ set $30 \mathrm{~cm}$ over the substrates, which were placed on a hot plate heated at $550^{\circ} \mathrm{C}$ (corresponding to a measured substrate surface temperature of $400^{\circ} \mathrm{C}$ ). An automatic syringe pump was used to deliver $1 \mathrm{~mL}$ of a solution containing $10 \mathrm{mM}$ of $\mathrm{Fe}(\mathrm{acac})_{3}(99.9+$, Aldrich) in $\mathrm{EtOH}(\geq 99.8 \%$ Fluka) to the spray head, every 30 seconds at a rate of $12 \mathrm{~mL} \mathrm{~min}^{-1}$ (spray length of $5 \mathrm{~s}$ ). The carrier gas (compressed air) flow, directing the spray to the substrates, was set to $15 \mathrm{~L} \mathrm{~min}^{-1}$. Four different amounts of liquid sprayed were tested: 20, 15, 8 and $6.5 \mathrm{~mL}$ (sample A, B, C and $\mathrm{D}$ respectively). After the spray, the samples were annealed for $5 \mathrm{~min}$ at $c a .500{ }^{\circ} \mathrm{C}$ before cooling to room temperature.

\section{Photoelectrochemical Cell Setup}

The photoelectrochemical cell configuration known as "cappuccino" was chosen to perform the electrochemical impedance analysis of the selected photoanodes. ${ }^{11}$ The cell was made of polyetheretherketone (Erta Peek $\left.{ }^{\circledR}\right)$ and had a $20 \mathrm{~mm}$ diameter uncoated fused silica window (Robson Scientific, England) pressed against an O-ring by a metallic window part. The cell was filled with an electrolyte aqueous solution of $1.0 \mathrm{M} \mathrm{NaOH}, \mathrm{pH}$ 13.6, at $25^{\circ} \mathrm{C}$ in which the hematite photoanode was immersed. The total immersed active surface area was approximately $2.5 \mathrm{~cm}^{2}, 0.5 \mathrm{~cm}^{2}$ of which being illuminated through an external mask. A $99.9 \%$ pure platinum wire (Alfa Aesar ${ }^{\circledR}$, Germany) was used as counter-electrode. The electrochemical measurements were performed using a standard three-electrode configuration. $\mathrm{An} \mathrm{Ag} / \mathrm{AgCl} / \mathrm{Sat} \mathrm{KCl}$ electrode (Metrohm, Switzerland) was used as reference electrode, the platinum wire as counter-electrode and the photoanode as working electrode.

\section{I-V Measurements}

The photocurrent-voltage $(I-V)$ characteristic curves were obtained applying an external potential bias to the cell and measuring the generated photocurrent using a ZENNIUM 
workstation (Zahner Elektrik, Germany) controlled by Thales software package (Thales $Z$ 1.0). The potential was reported against the reversible hydrogen electrode (RHE):

$E_{\mathrm{RHE}}=E_{\mathrm{AgCl}}+0.059 \mathrm{pH}+E_{\mathrm{AgCl}}^{\mathrm{o}}$, with $E_{A g C l}^{o}=0.1976 \mathrm{~V}$ at $25^{\circ} \mathrm{C}$.

The measurements were performed in the dark and under simulated sunlight, AM 1.5 G (150 W Xe lamp, $1000 \mathrm{~W} \mathrm{~m}^{-2}, 25^{\circ} \mathrm{C}$ ) calibrated with a c-Si photodiode, at a scan rate of $10 \mathrm{mV} \mathrm{s}^{-1}$ between $0.9 \mathrm{~V}_{\mathrm{RHE}}$ and $1.8 \mathrm{~V}_{\mathrm{RHE}}$. A standard three-electrode configuration was used with $1 \mathrm{M} \mathrm{NaOH}\left(\right.$ TitriPUR ${ }^{\circ}, 25^{\circ} \mathrm{C}, \mathrm{pH} 13.6$ ) as electrolyte, $\mathrm{Ag} / \mathrm{AgCl}$ sat. $\mathrm{KCl}$ as reference and a platinum wire as counter-electrode.

\section{EIS Measurements}

EIS measurements were performed using a ZENNIUM workstation. The frequency range was $0.01 \mathrm{~Hz}-100 \mathrm{kHz}$ and the magnitude of the modulation signal was $10 \mathrm{mV}$. All the measurements were performed at room temperature at an applied potential range of 0.9 $\mathrm{V}_{\mathrm{RHE}}-1.7 \mathrm{~V}_{\mathrm{RHE}}$ under dark conditions. The EIS spectra were fitted to the electrical analogue under study by means of the ZView ${ }^{\circledR}$ software.

\section{Results and discussion}

\section{Film Thickness}

UV-visible absorption data was used to estimate the thickness of the hematite samples. The absorbance spectra for all the selected photoanodes with different thicknesses are presented in Figure 1. They exhibit an absorption maximum around $400 \mathrm{~nm}$, followed by a gradual decrease in absorbance until $600 \mathrm{~nm}$. This wavelength corresponds to $2.06 \mathrm{eV}$, within the range of hematite band-gaps usually reported $(2.0-2.2 \mathrm{eV}) .{ }^{1}{ }^{10}$ Since there is a similarity in the shape of the spectra of all samples, their thickness can be estimated assuming a Lambertian absorption behaviour:

$\ln (1-A)=-\alpha \cdot \ell$

where $A$ is the absorbance, $\alpha$ is the hematite absorbance coefficient taken as $(44 \mathrm{~nm})^{-1}$ for a wavelength of $400 \mathrm{~nm}$ and $\ell$ is the thickness of the hematite film in nanometers. ${ }^{12}$. The absorbance spectra for all samples are presented in Figure 1. The thickness values obtained for the ultrathin-film hematite electrodes are reported in Table 1. 


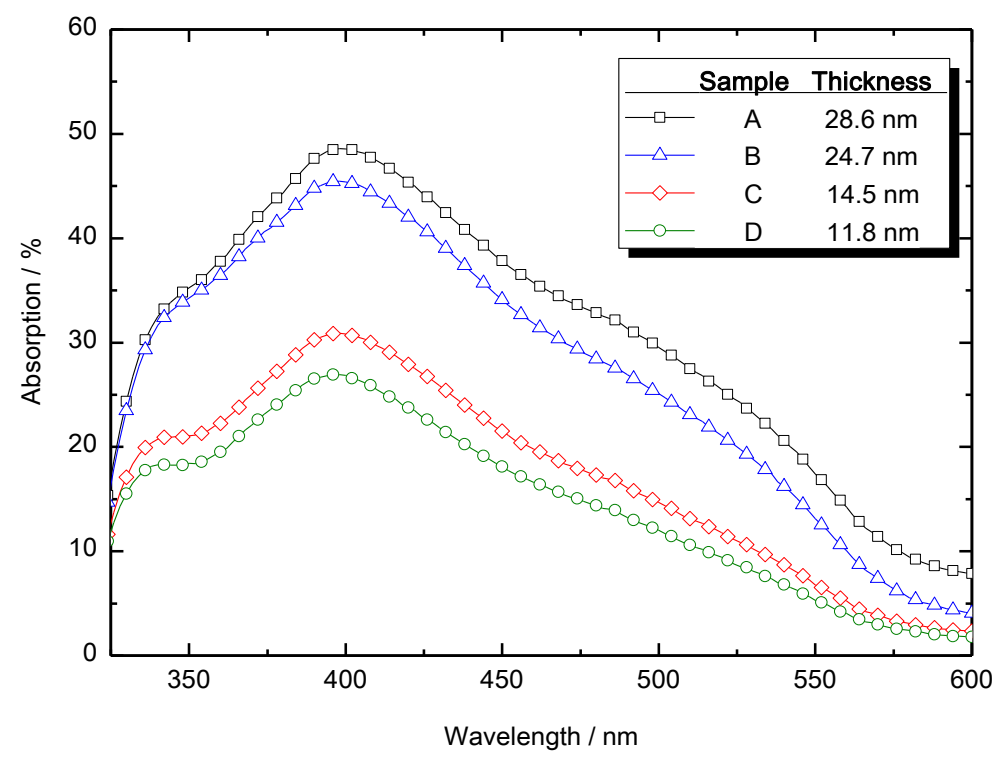

Figure 1. Absorption curves for the different samples; the absorbance was determined assuming a Lambertian relation.

\section{Photocurrent density-voltage characteristics}

The performance of hematite photoanodes with different thicknesses, obtained by sweeping current-potentiometry scans in $1 \mathrm{M} \mathrm{NaOH}$ electrolyte $(\mathrm{pH}$ 13.6) in the dark and under simulated solar illumination (AM $1.5 \mathrm{G}, \sim 100 \mathrm{~mW} \cdot \mathrm{cm}^{-2}, 25^{\circ} \mathrm{C}$ ), is shown in Figure 2. Performance details of the photoanode samples A to D are summarized in Table 1. Under illumination conditions (solid lines) it can be noticed that the thinnest hematite electrode (sample D) shows the lowest photoactivity. Moreover, by increasing the thickness of the photoanode the rising onset potential, for which the photocurrent density gradually increases with the applied potential, starts at more cathodic potentials. This means that the photoactivity properties of the hematite are enhanced by either the reduction of the overpotential for water oxidation, a shift in the flat-band potential, or the reduction of Fermi level. On the other hand, under dark conditions (dashed lines) the water oxidation onset potential, i.e. when the current in the dark sharply increases, of sample D arises well before the water oxidation onset potential of the other samples, whereas the thicker sample shows an onset of the dark current at more anodic potentials. When light is applied the same behaviour is observed: the water oxidation onset potential also starts at lower potentials for the thinnest sample. This phenomenon observed for the 
thinnest sample may be due to the presence of a TCO in direct contact with electrolyte in the semiconductor layer - see Figure 2.

Undoped hematite is a material with high electron-hole recombination rate and a low electronic mobility. Thus, the photoactive zone of this kind of photoelectrodes generally corresponds to the space charge layer. ${ }^{13}$ This means that photons absorbed deeper than this layer in the semiconductor film (bulk of the semiconductor) have very low probability to generate charges able to successfully reach the semiconductor surface and thus participate in photoreactions; actually the photogenerated holes will recombine with the electrons due to their short diffusion lengths. ${ }^{13}$ In order to reduce this recombination pathway, some authors suggested the use of very thin photoelectrodes. ${ }^{10,14}$ Thus, the film thickness can be adjusted to be in the same order of magnitude as the depletion layer, restricting in that way the recombination phenomenon. ${ }^{14}$ In these cases the excitation process happens in the depletion layer region and so the photoexcited electrons will efficiently reach the external circuit. On the other hand, if the excitation process occurs in the semiconductor bulk, recombination between the excited electrons and the corresponding holes takes place, followed by heat release. ${ }^{15}$ Consequently, for hematite material at a given applied potential, the photocurrent increases with the thickness of the semiconductor until reaching the space charge thickness. For semiconductor thicknesses above this critical value, the photocurrent is independent of the film thickness. ${ }^{13}$ The later explains why there are almost no differences in the maximum photocurrent values for samples A and B. Furthermore, for even thicker samples, an opposite effect responsible for a photocurrent drop may be observed: the increase of the thickness increases the electrical resistance in the semiconductor bulk. ${ }^{13}$ In fact, a $30 \mathrm{~nm}$ - thick sample was characterized in terms of its characteristic curves in the dark and under 1 sun illumination conditions producing a lower photocurrent density than the three thicker samples (A, B and C) - Figure S2 on the supporting info.

The difference in the photocurrent generated by samples $\mathrm{C}$ and $\mathrm{D}$ is greater than the one observed for samples A and B, even though they were prepared having approximately the same thickness step increment: samples A and B differ by $4 \mathrm{~nm}$ in thickness and show a photocurrent difference of just $0.02 \mathrm{~mA} \cdot \mathrm{cm}^{-2}$; on the other hand, from sample $\mathrm{D}$ to sample $\mathrm{C}$, an increase of also $4 \mathrm{~nm}$ in thickness originated an increase in photocurrent of about $0.10 \mathrm{~mA} \cdot \mathrm{cm}^{-2}$. This difference may be ascribed to factors like surface states and crystallinity, but also due to constraints imposed by the space charge layer thickness. Also, by increasing the sample's thickness, the strain in the hematite lattice (originated 
from the $\mathrm{FTO} /$ hematite interface) is more released at the hematite/electrolyte interface implying a better charge conduction in the lattice and then less bulk recombination and a better surface arrangement, responsible for a lower surface recombination. On the other hand, the huge difference in the water oxidation dark current onset for sample D can be also attributed to a higher TCO area exposed to the electrolyte. ${ }^{10}$ A sample with lower thickness was also produced and tested in terms of its $J$ - $V$ curves. Nevertheless, due to the large TCO area exposed to the electrolyte solution and the corresponding small amount of semiconductor material deposited over it, no significant differences are observed between the current produced under dark and under illumination conditions Figure $\mathrm{S} 2$ on the supporting info.

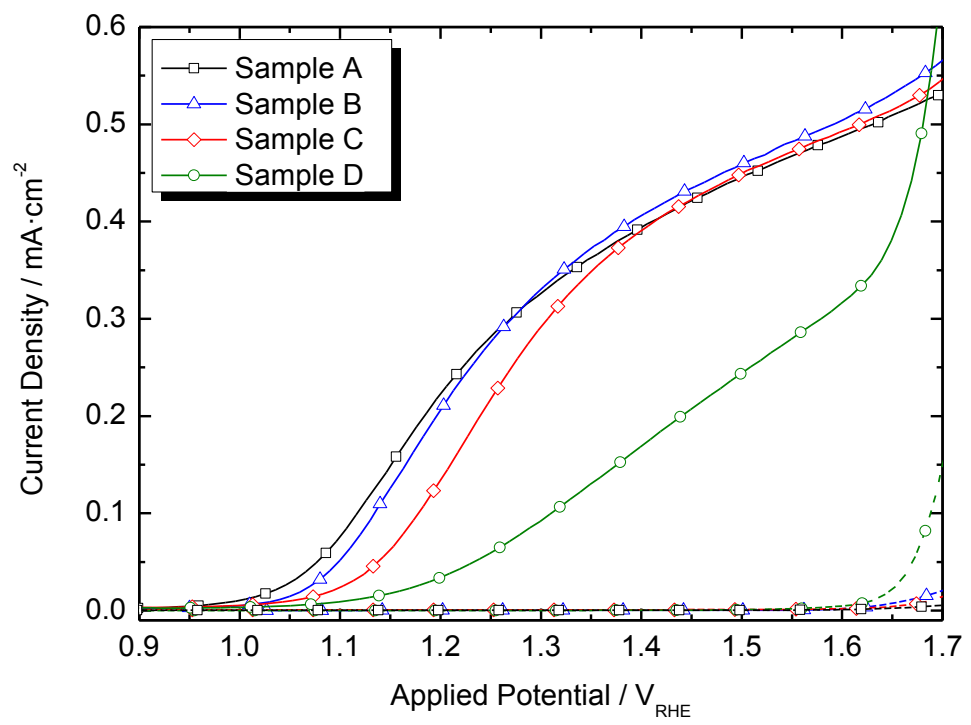

Figure 2. Photocurrent density-voltage characteristics of the prepared photoanode samples A to D in the dark (dashed lines) and under simulated 1 sun illumination $\left(100 \mathrm{~mW} \cdot \mathrm{cm}^{-2}\right.$, solid curves).

Table 1. Thicknesses and photocurrent-voltage performances of the ultra thin-film $\mathrm{Fe}_{2} \mathrm{O}_{3}$ photoanodes.

\begin{tabular}{|c|c|c|c|c|c|}
\hline \multirow[t]{2}{*}{ Sample } & \multirow[t]{2}{*}{$\begin{array}{l}\mathrm{Fe}_{2} \mathrm{O}_{3} \\
\text { thickness [nm] }\end{array}$} & \multicolumn{2}{|c|}{$\begin{array}{l}\text { Water oxidation onset potential } \\
\qquad\left[\mathrm{V}_{\mathrm{RHE}}\right]^{[\mathrm{a}]}\end{array}$} & \multicolumn{2}{|c|}{ Photocurrent density $\left[\mathrm{mA} \cdot \mathrm{cm}^{-2}\right]$} \\
\hline & & Dark & Light & (a) $1.23 \mathrm{~V}_{\mathrm{RHE}^{[b]}}$ & (a) $1.45 \mathrm{~V}_{\mathrm{RHE}^{[\mathrm{b}]}}$ \\
\hline A & 28.6 & 1.80 & 1.00 & 0.26 & 0.42 \\
\hline B & 24.7 & 1.66 & 1.03 & 0.25 & 0.43 \\
\hline $\mathrm{C}$ & 14.5 & 1.68 & 1.06 & 0.18 & 0.42 \\
\hline D & 11.8 & 1.62 & 1.14 & 0.05 & 0.21 \\
\hline
\end{tabular}




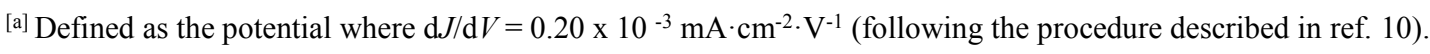

${ }^{[b]}$ At 1 Sun AM $1.5 \mathrm{G}\left(100 \mathrm{~mW} \cdot \mathrm{cm}^{-2}\right)$ in $1 \mathrm{M} \mathrm{NaOH}$.

\section{Electrochemical Impedance Spectroscopy measurements}

The electrochemical impedance measurements were performed using a three-electrode configuration. This configuration enables the detailed study of the electrochemical behaviour of the photoanode/electrolyte interface since the potential is measured with respect to a fixed reference potential, short-circuited with the counter-electrode. A negligible current is drawn through the reference electrode since very high impedance is observed in the device used to measure the potential difference between the working and reference electrodes; thus any change in the applied potential is reflected only in the working electrode. ${ }^{9,} 16$ Typically EIS data is analysed by means of equivalent electrical circuit models. Specifically in electrochemical systems such as PEC cells, fitting the experimental data to specific arrangements of electrical elements provides relevant information concerning charge transport, charge trapping and charge transfer at the interface of the photoelectrode/electrolyte. Nevertheless, the different preparation methods and the continuous progress in nanostructured photoelectrodes brought additional difficulties to a clear understanding of the charge transfer phenomena in the semiconductor/electrolyte interface. ${ }^{17}$ This interface was first rationalized in 1970 by Gerischer. ${ }^{18}$ When a n-type semiconductor comes into contact with an electrolyte containing a redox couple, a space charge layer arises in the semiconductor adjacent to the interface with the electrolyte due to the equilibration of the chemical potential - Figure $3 .{ }^{19}$ For the present study, ultrathin hematite samples prepared by spray pyrolysis method were used. This deposition method of $\mathrm{a}-\mathrm{Fe}_{2} \mathrm{O}_{3}$ from a solution of iron (III) acetylacetonate allowed obtaining uniform thin layers over large areas. ${ }^{10}$ 


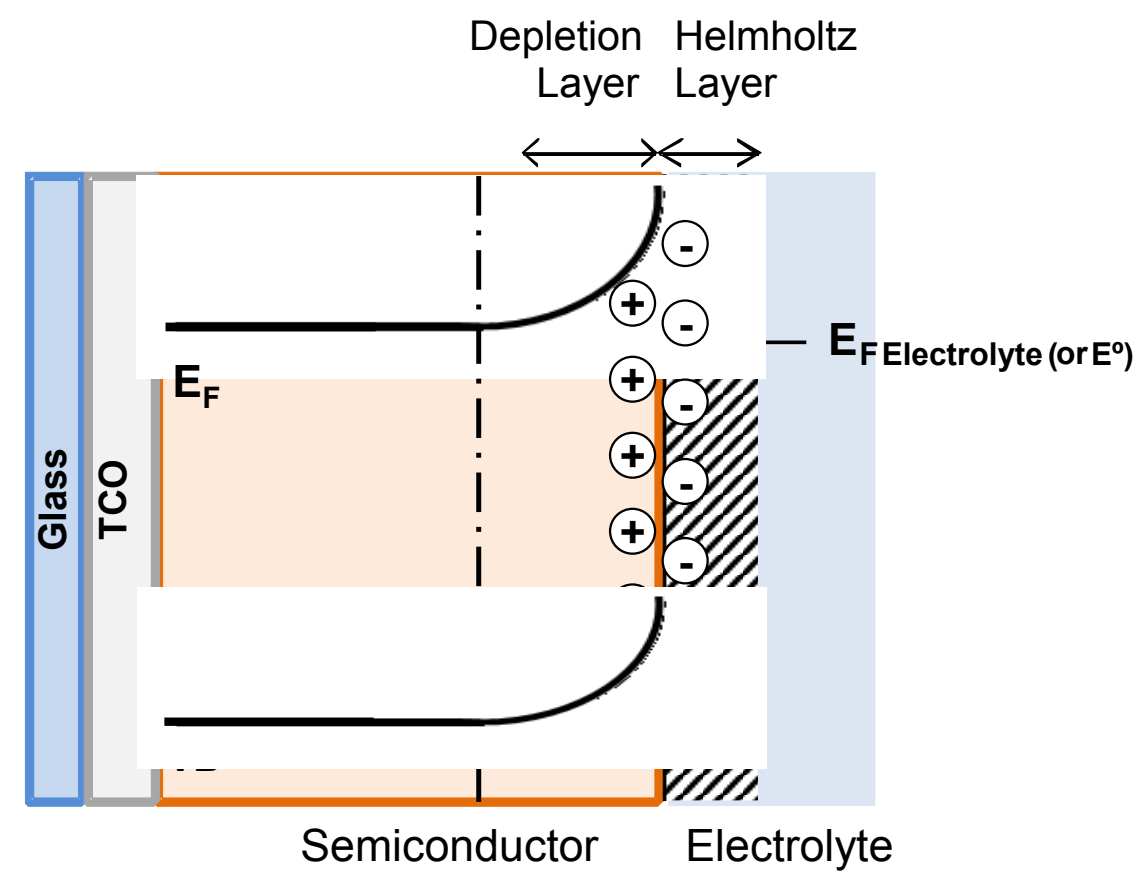

Figure 3. Energy diagram for an n-type semiconductor in charge-transfer equilibrium with the electrolyte.

Significant advances in applying EIS technique to hematite photoelectrodes have been made; for instance, alternative models explaining the two features observed in the EIS spectra can be found in the literature. ${ }^{2,5,7,20-23}$ Three different approaches for electrical analogues applied to PEC systems have been proposed ${ }^{17}$ : i) the classic Mott-Shottky relation, which relies on a simple RC circuit to describe the Helmholtz capacity constant fitted to the high frequencies range of the impedance spectra; ii) a more sophisticated circuit model that takes into account the trap states (i.e. surface states at low bias potential, bulk states far from the flat band); and finally iii) a model that considers a non-negligible Helmholtz capacitance due to the presence of a high surface area (nanostructure) or surface states. More recently, Bisquert et al. proposed an equivalent circuit which highlights the central role of surface states acting as recombination centres, trapping electrons from the conduction band and holes from the valence band under light conditions. ${ }^{23}$ One the other hand, under dark conditions the same authors considers a more simplified equivalent circuit - a Randles electrical circuit. They argue that since only one semi-circle is observed, then the space charge $\left(C_{S C}\right)$ and Helmholtz $\left(C_{H}\right)$ capacitances should be fitted as a single $C_{\text {Bulk }}$ capacitance. ${ }^{23}$

Even though only one semi-circle is observed for the system under study at given applied potentials, it is noticed that a Randles circuit does not fit the impedance spectra in all the frequency range. Thus, the electrical analogue sketched in Figure 4 is proposed for a thin 
layer semiconductor operating under dark conditions. Although this model does not give a phenomenological understanding about the charge transfer processes, it allows the quantification and identification of the resistive and capacitive elements that are related to the phenomena occurring in the semiconductor bulk and in the semiconductor/electrolyte interface. In this model the element $R_{\text {series }}$ is the series resistance, which includes the TCO resistance, the resistance related to the ionic conductivity in the electrolyte and the external contacts resistance (e.g. wire connections). The semiconductor/electrolyte interface can be modelled as a double capacitor, since the equilibrium process gives rise to a space charge layer - depletion layer - in the semiconductor side and to an electrical double layer of opposite sign in the electrolyte phase boundary - Helmholtz layer (Figure 3). The capacitance of the space charge region, $C_{\mathrm{SC}}$, and the Helmholtz capacitance, $C_{\mathrm{H}}$, can be of the same order of magnitude and none should be neglected when fitting the EIS data. ${ }^{22}$ The proposed electrical analogue comprises two RC elements in series representing the semiconductor bulk and the surface phenomena - Figure $4 .{ }^{24}$ According to this, and bearing in mind that the electronic processes in the bulk are generally faster than the charge transfer processes or diffusion of ions in solution, the low-frequency response was assigned to the semiconductor/electrolyte charge transfer resistance, $R_{\mathrm{CT}}$, together with $C_{\mathrm{H} .}{ }^{17,24}$ Thus, the fast electronic processes, high-frequency range $(100 \mathrm{~Hz}$ to $1 \mathrm{kHz})$, are assigned to the events occurring in the semiconductor bulk, which are described by the resistance, $R_{\mathrm{SC}}$, and the space charge capacitance, $C_{\mathrm{SC}}$. In fact, this $\mathrm{RC}$ element is the combination of different resistances and capacitances related to the transport in the semiconductor layer, the charge diffusion in the space charge layer and surface trap charging by electrons and holes, which have similar time constants, independent of the applied potentials. 


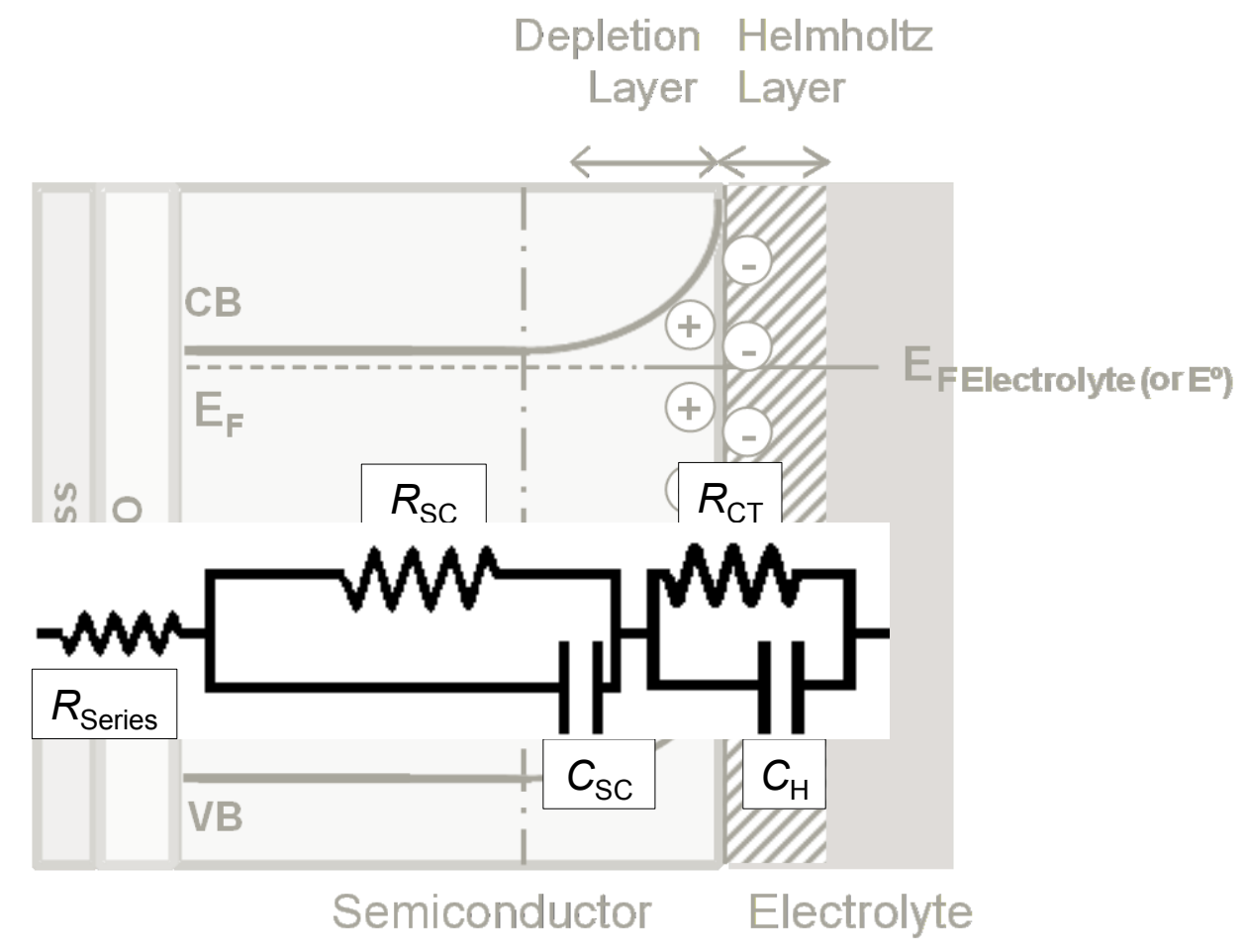

Figure 4. Energy diagram of semiconductor/electrolyte interface with the corresponding electrical circuit analogue.

In cells formed by nanostructured semiconductors, capacitors do not behave ideally. Instead they are often found to act like constant phase elements (CPE), non-ideal capacitances associated with a non-uniform distribution of current in the heterogeneous materials. The impedance of a CPE is defined as:

$Z_{C P E}=\frac{1}{j \omega C^{n}}$

where $n(0<n<1)$ is an empirical constant. This parameter was kept between 0.7 and 1 . 9,25

The impedance measurements of the four different samples under study were carried out, under dark conditions, in the potential range of $0.9 \mathrm{~V}_{\mathrm{RHE}}$ to $1.7 \mathrm{~V}_{\mathrm{RHE}}$, with a step of 50 $\mathrm{mV}$. Fitting the electrical circuit analogue to the experimental EIS data, parameters $R_{\mathrm{SC}}$, $R_{\mathrm{CT}}, C_{\mathrm{SC}}$ and $C_{\mathrm{H}}$ were obtained. These parameters are plotted in Figure 5a) $-\mathrm{d}$ ) as a function of the applied potential.

Figure 5a) plots the high frequency resistance $R_{\mathrm{SC}}$ related to the electron transport in the bulk and in the space charge of the photoanode as a function of the applied potential. Increasing the thickness of the semiconductor, higher electron transport resistances are 
achieved. Between 0.9 and $1.4 \mathrm{~V}_{\mathrm{RHE}}$ the electron transport resistance $R_{\mathrm{SC}}$ is almost independent of the bias voltage applied for all thicknesses considered; this is in straight agreement with the $J-V$ characteristics under dark conditions (Figure 2), which show almost no net current flow until reaching 1.4 $\mathrm{V}_{\text {RHE. }}$ In fact, when the applied potential is further increased (for values higher than $1.4 \mathrm{~V}$ RHE) the semiconductor Fermi level becomes closer to the valence band than to the conduction band near the semiconductor surface; thus an inversion layer is formed. Near the surface, the n-type semiconductor has a p-type behaviour (the holes locally become the majority charge carrier). When the Fermi level crosses the valence band edge due to the high applied potential, the formation of a deep depletion layer is then observed and the holes can react with the hydroxyl ions in the solution. ${ }^{13}$ At approximately $1.6 \mathrm{~V}_{\text {RHE }}$, Figure 5a) starts exhibiting a steeply decrease in the bulk resistance, $R_{S C}$. This potential coincides with the onset of the water oxidation dark current, enabling efficient hole reaction with the oxidized species in the electrolyte solution. For sample D, the thinnest photoanode, this steeply decrease of the bulk semiconductor resistance takes place earlier, suggesting that the TCO on sample D is not fully covered with $\mathrm{Fe}_{2} \mathrm{O}_{3}$ material as discussed elsewhere. ${ }^{10}$ 

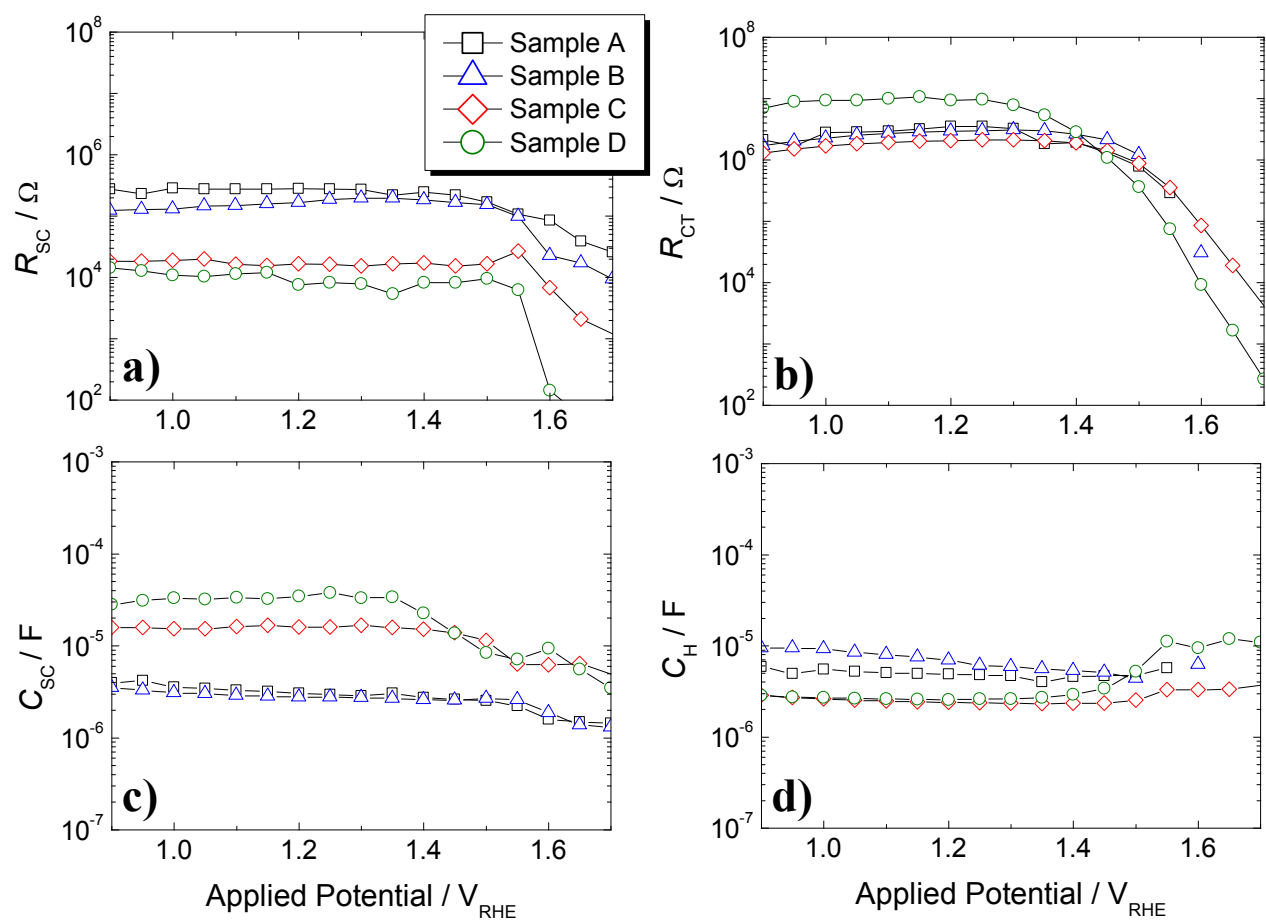

Figure 5. Impedance results obtained by fitting the experimental data to the equivalent electrical circuit shown in Figure 4 plotted versus the applied potential with reference to RHE: a) Bulk semiconductor resistances; b) Charge transfer resistance; c) Space charge capacity; d) Helmholtz capacity.

Figure $5 \mathrm{~b})$ shows the charge transfer resistances $\left(R_{\mathrm{CT}}\right)$ in the semiconductor/electrolyte interface ascribed to the low frequencies range in Nyquist diagrams for the four samples. $R_{C T}$ values are larger than the bulk resistances $R_{\mathrm{SC}}$ (Figure $5 \mathrm{~b}$ ) for the entire voltage range, i.e. $R_{\mathrm{CT}} \geq 10^{6}$ between $0.9 \mathrm{~V}_{\mathrm{RHE}}$ and $1.4 \mathrm{~V}_{\mathrm{RHE}}$. In addition, it is visible in Figure $5 \mathrm{~b}$ ) that the charge transfer resistance in the semiconductor/electrolyte interface for the three thicker samples (samples A, B and C) is almost independent of the thickness. This behaviour is in agreement with the similar characteristic curves exhibited by these samples - Figure 2. Moreover, $R_{C T}$ values remain constant up to $1.6 \mathrm{~V}_{\mathrm{RHE}}$, value at which the resistances start to decrease. The thinnest sample (sample D) also follows this behavior but exhibits a charge transfer resistance in the interface one order of magnitude higher. This may indicate the existence of a photoinactive "dead" layer in the interface $\mathrm{TCO} /$ semiconductor or possible due to a bad surface arrangement at the $\mathrm{Fe}_{2} \mathrm{O}_{3} /$ electrolyte interface. ${ }^{26}$ The differences observed on the photoanode D performance can also be related to its lower crystallinity and higher trap states concentration, but those contributions would be more evident under a light impedance spectroscopy 
measurement. ${ }^{10,26}$ Nevertheless, other two causes may explain this low performance: $i$ ) the TCO layer can be in contact with the electrolyte solution due to a non-uniform hematite deposition, which is responsible for the earlier water oxidation onset potential; or $i$ ) the depletion layer thickness is limited by the semiconductor film thickness, $c a$. of $11 \mathrm{~nm}$. Thus, the depletion layer width of sample D is thinner than the depletion layer of the other samples and of the same order of magnitude of the whole film thickness. This induces a smaller electric field in the depletion layer region, restricting the efficiency of charges separation.

As mentioned before, when a semiconductor electrode is brought into contact with an electrolyte solution, the two phases evolve to reach the electrochemical equilibrium. ${ }^{3,19}$, ${ }^{27}$ If the redox potential of the solution and the Fermi level of the semiconductor do not lie at the same potential energy, a movement of charges between the semiconductor and the solution is required in order to equilibrate the two phases. ${ }^{3}$ The excess of charges positioned on the semiconductor side is not located at its surface, as it would be in the case of a metallic electrode, but instead it extends in the electrode for a deeper distance space charge layer. Since the majority charge carriers of the semiconductor are removed from this region, it may also be referred to as a depletion region. ${ }^{28}$ This layer, depleted of majority carriers, is compensated by a charge layer of opposite sign that is induced in the electrolyte side, the so called Helmholtz layer - formed by oriented water molecules dipoles and electrolyte ions adsorbed at the electrode surface. ${ }^{3,27}$ Thus, two capacitances can be distinguished, namely, the space charge capacitance, $C_{\mathrm{SC}}$, and the Helmholtz capacitance, $C_{\mathrm{H}}$, representing the phenomena occurring at the interface semiconductor/electrolyte. ${ }^{29}$ Figure $5 \mathrm{c}$ ) and d) plots $C_{\mathrm{SC}}$ and $C_{\mathrm{H}}$, respectively, as a function of the applied bias. The space charge capacitance $\left(C_{\mathrm{SC}}\right)$ increases as the film thickness decreases, with the exception of Sample A, which presents the same $C_{\mathrm{SC}}$ value as Sample B - Figure 5c). Moreover, $C_{\mathrm{SC}}$ is almost independent of the applied potential up to $1.4 \mathrm{~V}_{\text {RHE}}$. For higher applied potentials, the water oxidation onset potential is achieved and the minority carriers in the depletion layer react with the ions in the electrolyte, consequently the capacitance decreases. The later effect is more visible with samples $\mathrm{C}$ and $\mathrm{B}$. For samples $\mathrm{A}$ and $\mathrm{B}$ a slightly decrease on $C_{\mathrm{SC}}$ values is noticed at all applied potentials, this effect may be due to the increase of the depletion layer width. ${ }^{24}$ In what concerns the Helmholtz capacitance $C_{\mathrm{H}}$ - Figure 5d) - a different behaviour is observed. The Helmholtz double layer width is generally smaller than the width of the depletion layer ${ }^{19}$ and, consequently, the capacitance associated to the Helmholtz layer is 
normally higher. ${ }^{3}$ Indeed, in the present study this is only true, i.e. $C_{H}>C_{S C}$, for the two thicker samples (sample A and B). The later behaviour is not observed with the two thinner samples, probably because the depletion layer thickness of the thinnest samples is somehow limited by the semiconductor thickness. Even though the space charge capacitance related to very thin films is not yet fully understood, one possible reason for this behaviour may be related to the way that charges are distributed within the space charger layer in thicker photoanodes. ${ }^{24}$ As mentioned, the capacitance values of $C_{\mathrm{H}}$ remain almost constant up to $1.4 \mathrm{~V}_{\mathrm{RHE}}$ when the water oxidation onset potential is reached. For higher applied voltages, the capacitance values related to the Helmholtz layer slightly increase due to the movement of the majority carriers leaving this layer. Further insights can be obtained through Mott-Schottky analysis - Equation (4):

$$
\frac{1}{C_{\mathrm{SC}}^{2}}=\frac{2}{\varepsilon_{0} \varepsilon_{\mathrm{r}} e N_{\mathrm{D}} A^{2}}\left(\left(V_{\mathrm{E}}-V_{\mathrm{FB}}\right)-\frac{k T}{e}\right)
$$

where, $\varepsilon_{\mathrm{r}}$ is dielectric constant of the semiconductor (assumed 80 for undoped hematite $)^{30}, \varepsilon_{0}$ is the permittivity of space, $k$ is the Boltzmann's constant, $T$ the absolute temperature, $e$ is the elementary charge, $N_{\mathrm{D}}$ is the donor density and $V_{\mathrm{E}}$ and $V_{\mathrm{FB}}$ are the applied voltage and the flat band potentials, respectively. By plotting $1 / \mathrm{C}_{\mathrm{SC}}^{2}$ as a function of the applied bias potential, a straight line should be obtained; the donor density, $N_{\mathrm{D}}$, can then be determined from the slope of this line, while the flat band potential, $V_{\mathrm{FB}}$, value can be obtained by extrapolating the interception of the straight line with the axis of the applied potential -(4). However, for using the Mott-Schottky model two main assumptions must be guaranteed: i) the two capacitances, in the space charge region and in the double layer, can only be considered together if the space charge capacitance is much smaller than the double layer capacitance (2-3 orders of magnitude) and, consequently, the contribution of the double layer capacitance to the total capacitance would be negligible; and ii) the Mott-Schottky model is adequate provided the frequency is high enough, in the order of $\mathrm{kHz} .{ }^{15}$ Having in mind these assumptions, it is visible that the thinnest samples, $\mathrm{C}$ and $\mathrm{D}$, do not fulfil all the requirements, mainly the point $\mathrm{i}$ ): $C_{H}>C_{S C}$, indicating that other factors may be interfering on the EIS response; when trying to plot $1 / \mathrm{C}_{\mathrm{SC}}^{2}$, the obtained results are horizontal lines (zero slope)-Figure 6 . Two different processes can be responsible for this behaviour: i) the semiconductor thickness 
is limiting the space charge capacitance, or/and ii) TCO exposition to the electrolyte, which imposes a new capacitive element that can be observed under dark conditions. ${ }^{31}$

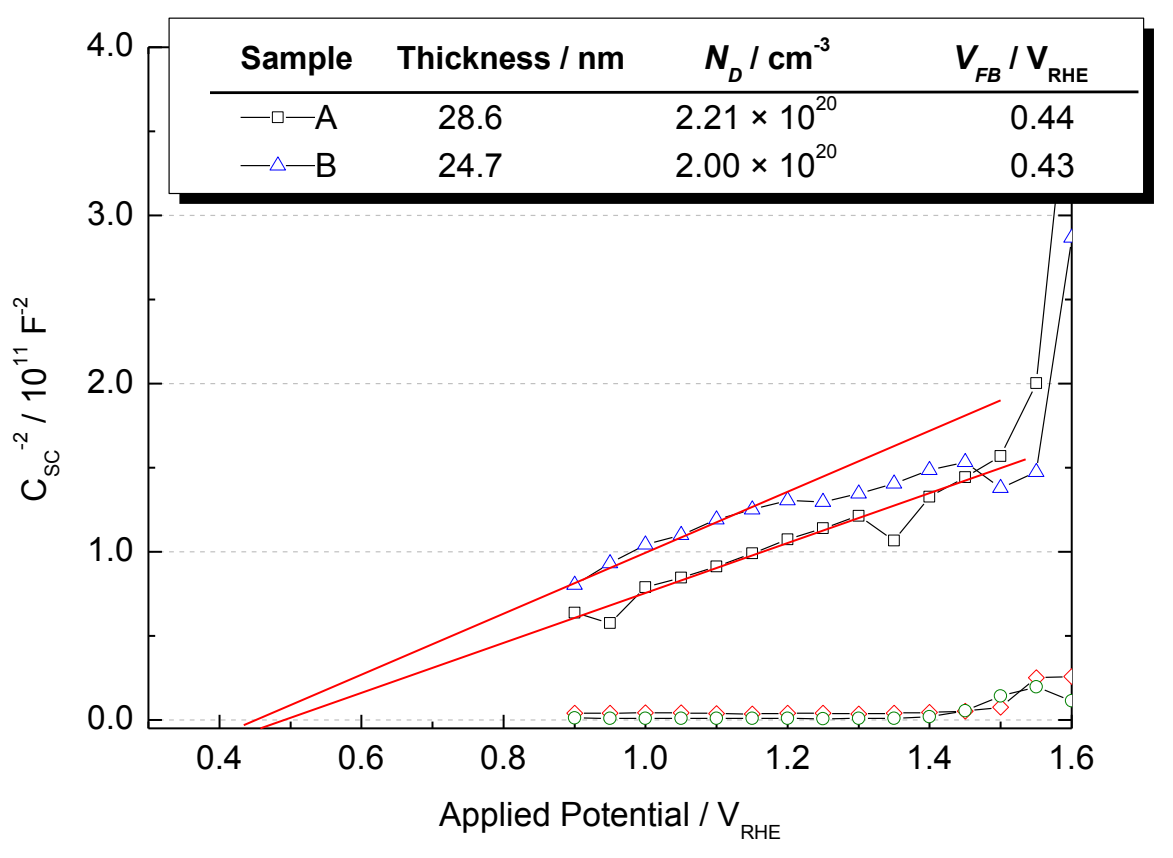

Figure 6. Mott-Schottky plots calculated from $C_{S C}$ values determined from fitting the impedance spectra for all range of applied bias potential. The inset table shows the obtained values for donor density and flat band potential determined from the slop of $1 / C_{\mathrm{SC}}^{2}$ vs. applied potential and by the extrapolation of $1 / C_{\mathrm{SC}}^{2}=0$, respectively.

The Mott-Schottky model was then applied to the two thicker samples under study, A and $\mathrm{B}$, for the applied potentials range between 0.7 and $1.4 \mathrm{~V}_{\mathrm{RHE}}$, which corresponds to a linear behaviour - Figure 6 . The calculated values of donor density regarding the two thicker samples are in line with the ones reported in literature for doped hematite structures $-N_{\mathrm{D}} \sim 10^{20} \mathrm{~cm}^{-3} \cdot{ }^{30,32,33}$ Even though, undoped structures are being analysed, these values still may be acceptable bearing in mind that the pre-treatment with TEOS before spraying the thin $\mathrm{Fe}_{2} \mathrm{O}_{3}$ layer is in some way acting as a doping agent, increasing the donor density values. Moreover, applying the Mott-Schottky model to a sample with $28.5 \mathrm{~nm}$-tick, the obtained value of donor density was $\sim 10^{18} \mathrm{~cm}^{-3}$, confirming that are the TEOS-pretreated substrates that confers to the ultra-thin hematite samples slightly doped characteristics. The obtained flat band potentials for samples A and B are $0.44 \mathrm{~V}_{\mathrm{RHE}}$ and $0.43 \mathrm{~V}_{\mathrm{RHE}}$, respectively.

The samples behaviour suggests that the best thickness for this type of electrodes is somewhere limited around the $20 \mathrm{~nm} .{ }^{34}$ For lower thicknesses, the space charge layer 
width and consequently the photo-response is limited by the semiconductor thickness and thus the photocurrent generated is lower.

Indeed, correlating the samples thickness differences with their electrochemical impedance spectroscopy response, it was possible to discriminate the two main phenomena occurring on semiconductor/electrolyte interfaces of photoelectrochemical systems under dark conditions: the space charge layer in the semiconductor side depletion layer; and the electrical double layer of opposite sign in the electrolyte phase Helmholtz layer. These observations are of great interest since the majority of electrical analogues suggested for these systems consider those layers together. This is only true under light conditions since it is not possible to individualize both responses in the EIS spectrum. Moreover, using the proposed electrical analogue to the $30 \mathrm{~nm}$-thick sample (Figure S3 on the supporting info) it was possible to conclude that the semiconductor bulk/depletion layer resistance $\left(R_{\mathrm{SC}}\right)$ is probably controlling the performance of the sample since it increases with the photoelectrode thickness.

Figure 7 plots the normalized values of resistance and capacitance for an applied potential of $1.25 \mathrm{~V}_{\mathrm{RHE}}$ as a function of the semiconductor layer thickness. In terms of resistances, sample $\mathrm{C}$ shows the best value when compared with the other three samples. Again, in terms of capacitances values they are somehow inconclusive since $C_{\mathrm{H}}$ is lower than $C_{\mathrm{SC}}$ for sample $\mathrm{C}$ and $\mathrm{D}$. 

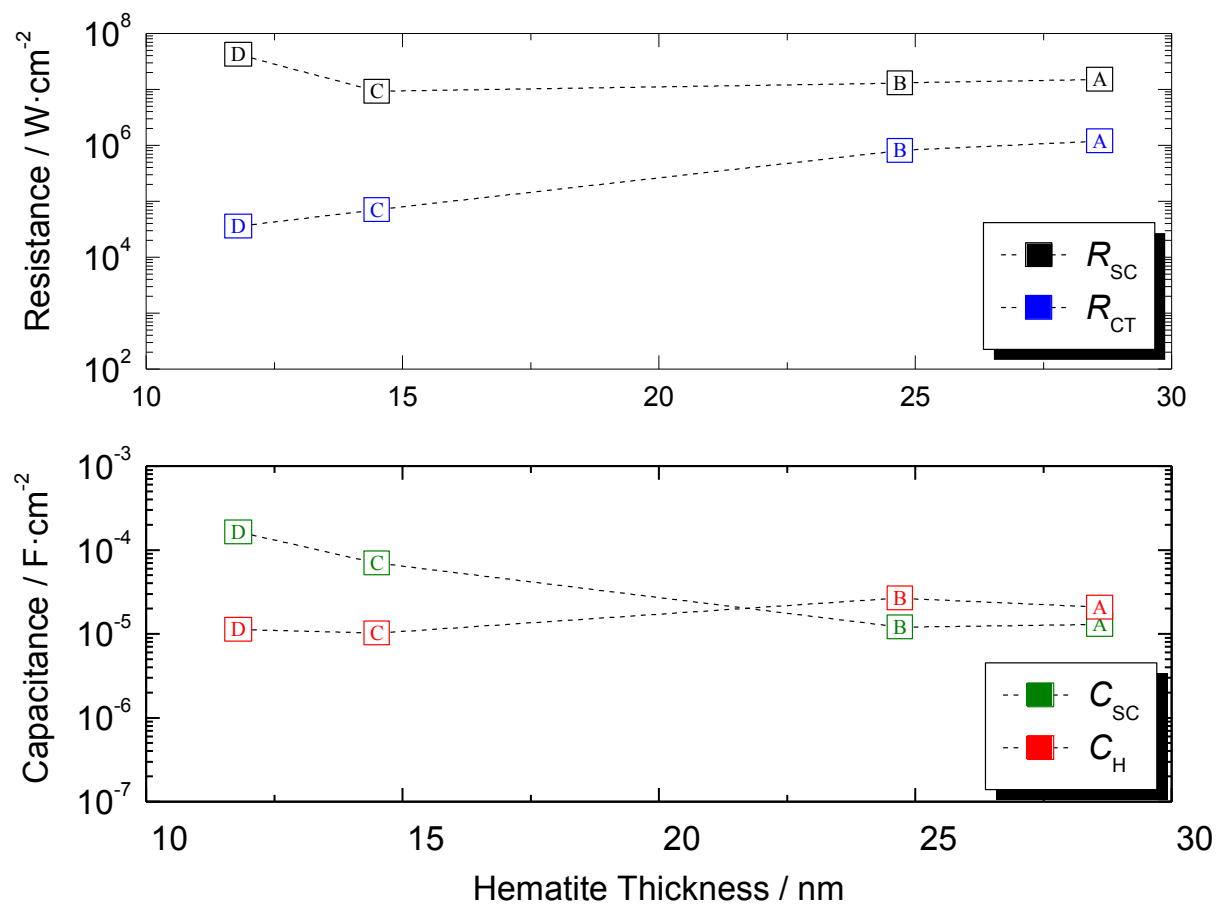

Figure 7. Comparison between the normalized resistance and capacitance values obtained for the four different samples under study at $1.25 \mathrm{~V}_{\mathrm{RHE}}$ applied voltage. Symbols $\mathrm{A}$ B $\mathrm{C}$ and $\mathrm{D}$ represents sample A, B, C and D, respectively.

As mentioned, the fact of $C_{\mathrm{H}}$ being lower than $C_{\mathrm{SC}}$ may indicate the existence of TCO exposed to the electrolyte and consequently, other capacitive elements can appear. In fact, SEM analysis and the corresponding energy-dispersive X-ray spectroscopy (EDS) was performed to prove the presence of these uncovered zones in the sample with smaller thickness. In Figure 8a) two areas with different colorations are clearly identified: a lighter zone marked as Z1 and a darker zone marked as Z2. These zones were subjected to a more detailed analysis by EDS, which shows that Z1 zone has a lower quantity of iron when compared with the darker area Z2 - Figure 8b). This undoubtedly indicates the absence of hematite material in Z1 zone, thus implicating TCO exposure. These analyses were performed for the other samples under study - A, B and D; however, a large exposed area of TCO was only identified in sample D. 


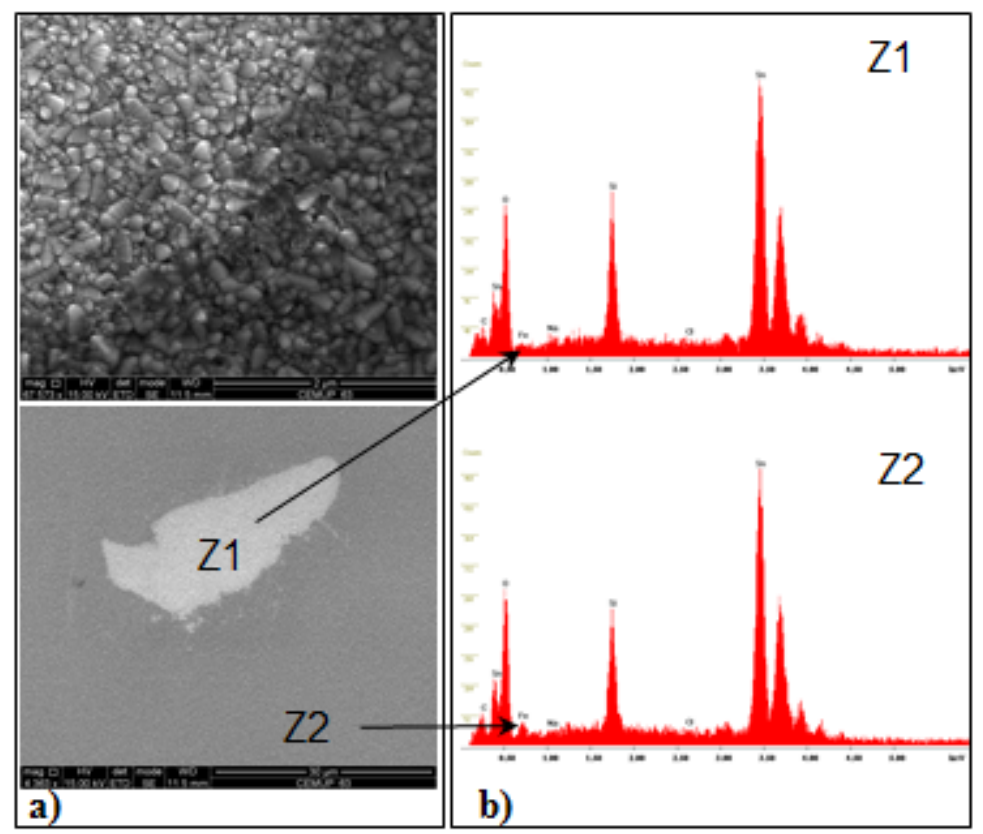

Figure 8. a) SEM analysis of the exposed TCO on sample D $(10.6 \mathrm{~nm})$ and b) the respective EDS analysis related to the two marked zones, $\mathrm{Z} 1$ and Z2.

\section{Conclusions}

The present work studies the electrochemical behaviour of four samples with different thicknesses under dark conditions by electrochemical impedance spectroscopy. The impedance spectra obtained were fitted to a simple electrical analogue describing the phenomena occurring in the semiconductor/electrolyte interface as well as in the semiconductor bulk/depletion layer was proposed and discussed. For validation purposes of the electrical analogue, four samples of $\alpha$-hematite prepared by spray pyrolysis with different thicknesses (11.8, 14.5, 24.7 and $28.6 \mathrm{~nm})$ were used. All $\alpha$-hematite photoanodes showed significant electron transport limitations, producing lower photocurrent densities than the ones reported in literature; the best performing sample sample A - showed an onset potential at 1.09 $\mathrm{V}_{\mathrm{RHE}}$ and a photocurrent plateau of about $0.4 \mathrm{~mA} \cdot \mathrm{cm}^{-2}$ at an applied bias of $1.45 \mathrm{~V}$ RHE. The proposed electrical analogue considers a series resistance with two $\mathrm{RC}$ circuits also in series: $R_{\mathrm{SC}}$ and $C_{\mathrm{SC}}$, which characterize the semiconductor bulk/depletion layer resistance and the space charge capacitance, respectively; $R_{\mathrm{CT}}$ and $C_{\mathrm{H}}$, represent the charge transfer at semiconductor/electrolyte interface and the Helmholtz capacitance, respectively. The electrochemical impedance spectroscopy analysis showed relevant information about the major internal charge 
transfer resistances, as well as the main charge accumulation zones in the photoelectrodes and in the semiconductor/electrolyte interface, under dark conditions. Moreover, with the EIS data was possible to identify and individualize the two different layers, responding at different frequencies, by using photoelectrodes with different thicknesses. Even though the thicker photoelectrodes showed higher semiconductor resistances, they exhibited lower charge transfer resistances values in the interface with the electrolyte, resulting in better performances; this effect may be explained by higher electrical fields exhibited by the thicker samples (lower $C_{\mathrm{SC}}$ ). The space charge and charge transfer resistances obtained by EIS technique provide relevant information to determine the optimal sample thickness. Moreover, for the two thinnest samples, it was observed that the space charge layer width and consequently the photo-response were limited by the semiconductor thickness. Additionally, it was also identified areas of TCO not coated with hematite and contacting directly with the electrolyte. This direct contact affected the EIS response and originated deviations to the proposed electrical analogue.

Nevertheless, the proposed electrical analogue addresses quite well the EIS spectra and a very good agreement were observed between fitted and experimental data. While this model fits well to $\alpha$-hematite photoelectrodes prepared by spray pyrolysis under dark conditions, it has yet to be verified its applicability to doped structures. The presence of two difference zones is shown through the use of two $\mathrm{RC}$ circuit elements under dark conditions. However, when illuminated other contributions may arise being necessary to apply other elements to the analogue circuit to fully characterize the PEC system.

\section{Acknowledgment}

T. Lopes and L. Andrade are grateful to the Portuguese Foundation for Science and Technology (FCT) for their $\mathrm{PhD}$ and Post-Doc fellowship, (references: SFRH/BD/62201/2009 and SFRH/BPD/74944/2010, respectively). Thanks to the European Commission for funding (Project NanoPEC - Nanostructured Photoelectrodes for Energy Conversion; contract number 227179). The authors also thank Dr. Thomas Moehl for useful discussions.

\section{Supporting Information Description}

The Nyquist spectra for three different applied potentials for each sample under study are shown in the support information. 
To validate the proposed electrical analogue two more photoelectrodes with the thicknesses of $7 \mathrm{~nm}$ and $30 \mathrm{~nm}$ were prepared and characterized. The obtained results are shown in the supporting information.

\section{References}

1. R. v. d. Krol, Y. Liang and J. Schoonman, Journal of Materials Chemistry, 2008, 18, 2311-2320.

2. V. M. Aroutiounian, V. M. Arakelyan, G. E. Shahnazaryan, G. M. Stepanyan, E.

A. Khachaturyan and J. A. Turner, Comptes Rendus Chimie, 2006, 9, 325-331.

3. A. J. Nozik, Annual Review of Physical Chemistry, 1978, 29, 189-222.

4. A. Fujishima and K. Honda, Nature, 1972, 238, 37-38.

5. V. M. Aroutiounian, V. M. Arakelyan, G. E. Shahnazaryan, H. R. Hovhannisyan, H. Wang and J. A. Turner, Solar Energy, 2007, 81, 1369-1376.

6. A. J. Bard and L. R. Faulkner, Electrochemical methods fundamentals and applications, John Wiley \& Sons, New York, 1980.

7. V. M. Aroutiounian, V. M. Arakelyan, G. E. Shahnazaryan, G. M. Stepanyan, J. A. Turner and S. S. Kocha, Electrochimica Acta, 2000, 45, 1999-2005.

8. V. M. Aroutiounian, V. M. Arakelyan, G. E. Shahnazaryan, G. M. Stepanyan, J. A. Turner and O. Khaselev, International Journal of Hydrogen Energy, 2002, 27, 33-38.

9. T. Lopes, L. Andrade, H. A. Ribeiro and A. Mendes, International Journal of Hydrogen Energy, 2010, 35, 11601-11608.

10. F. Le Formal, M. Grätzel and K. Sivula, Advanced Functional Materials, 2010, 20, 1099-1107.

11. I. Cesar, A. Kay, J. A. Gonzalez Martinez and M. Gratzel, Journal of the American Chemical Society, 2006, 128, 4582-4583.

12. L. A. Marusak, R. Messier and W. B. White, Journal of Physics and Chemistry of Solids, 1980, 41, 981-984.

13. A. Duret, EPFL, 2005.

14. K. Itoh and J. O. M. Bockris, Journal of Applied Physics, 1984, 56, 874-876.

15. A. W. Bott, Current Separations, 1998, 17.

16. R. v. d. Krol and M. Grätzel, Photoelectrochemical Hydrogen Production, Springer US, 2012.

17. K. Sivula, F. Le Formal and M. Grätzel, ChemSusChem, 2011, 4, 432-449. 
18. H. Gerischer, Semiconductor Electrochemistry, in Treatise on Physical Chemistry Vol. IXA: Electrochemistry, 1970, 463-542.

19. A. Aruchamy, G. Aravamudan and G. Subba Rao, Bulletin of Materials Science, 1982, 4, 483-526.

20. N. S. McAlpine and R. A. Fredlein, Journal of Electroanalytical Chemistry and Interfacial Electrochemistry, 1988, 252, 61-69.

21. J. van de Lagemaat, N. G. Park and A. J. Frank, The Journal of Physical Chemistry $B, 2000,104,2044-2052$.

22. J. Wielant, V. Goossens, R. Hausbrand and H. Terryn, Electrochimica Acta, 2007, 52, 7617-7625.

23. B. Klahr, S. Gimenez, F. Fabregat-Santiago, T. Hamann and J. Bisquert, Journal of the American Chemical Society, 2012, 134, 4294-4302.

24. F. Le Formal, N. Tetreault, M. Cornuz, T. Moehl, M. Gratzel and K. Sivula, Chemical Science, 2011, 2, 737-743.

25. E. Barsoukov and J. R. Macdonald, Impedance spectroscopy: theory, experiment, and applications, 2nd edn., Wiley-Interscience, Hoboken, N.J., 2005.

26. K. Sivula, F. L. Formal and M. Grätzel, Chemistry of Materials, 2009, 21, 28622867.

27. T. Bak, J. Nowotny, M. Rekas and C. C. Sorrell, International Journal of Hydrogen Energy, 2002, 27, 991-1022.

28. M. Chirita and I. Grozescu, Chemical Bulletin "POLITEHNICA" University of Timisoara, 2009, 54, 1.

29. M. A. Butler and D. S. Ginley, Journal of Materials Science, 1980, 15, 1-19.

30. I. Cesar, K. Sivula, A. Kay, R. Zboril and M. Grätzel, The Journal of Physical Chemistry C, 2008, 113, 772-782.

31. F. Fabregat-Santiago, G. Garcia-Belmonte, J. Bisquert, A. Zaban and P. Salvador, The Journal of Physical Chemistry B, 2001, 106, 334-339.

32. T. Lindgren, L. Vayssieres, H. Wang and S.-E. Lindquist, in Chemical Physics of Nanostructured Semiconductors, eds. A. I. Kokorin and D. W. Bahnemann, VSP - An imprint of BRILL, Boston, Editon edn., 2003.

33. S. U. M. Khan and J. Akikusa, The Journal of Physical Chemistry B, 1999, 103, 7184-7189.

34. B. M. Klahr, A. B. F. Martinson and T. W. Hamann, Langmuir, 2010, 27, 461468. 